\title{
Suppressing Dark Current in Organic Phototransistors Through Modulating Electron Injection via a Deep Work Function Electrode
}

Ren Shidachi, Hiroaki Jinno, Sunghoon Lee, Tomoyuki Yokota, Takao Someya*

Department of Electrical Engineering and Information Systems,

The University of Tokyo

7-3-1 Hongo, Bunkyo-ku

Tokyo 113-8656, Japan

Phone: +81- 3-5841-0411 E-mail: someya@ee.t.u-tokyo.ac.jp 
(a)

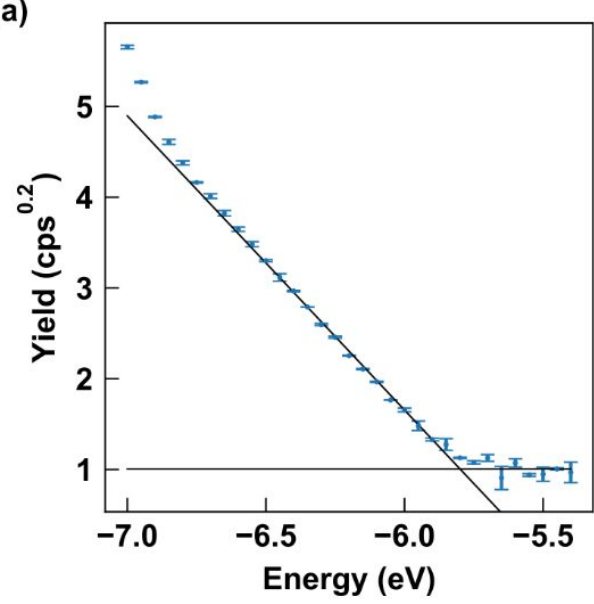

(b)

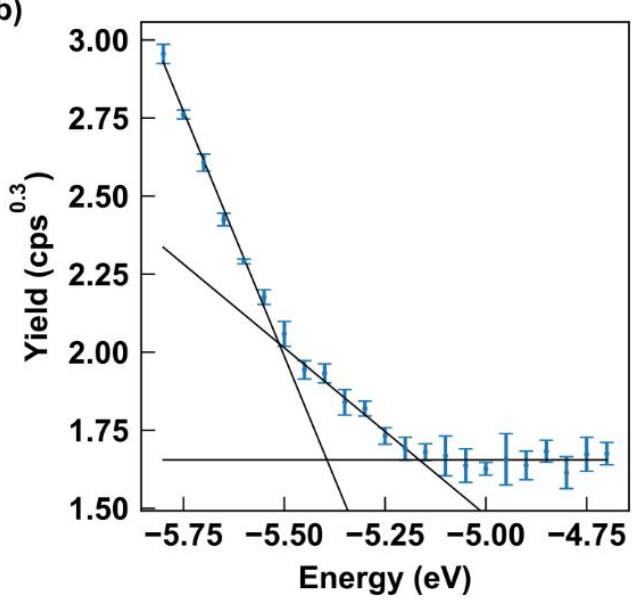

Figure. S1 The UPS results. (a) UPS result for $\mathrm{MoO}_{\mathrm{x}}$ (b) UPS results for $\mathrm{OC}_{1} \mathrm{C}_{10}$-PPV:PCBM. 
(a)

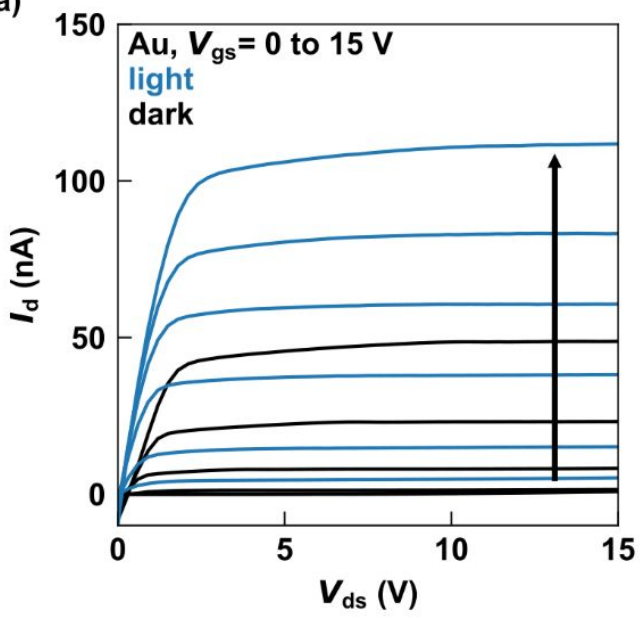

(b)

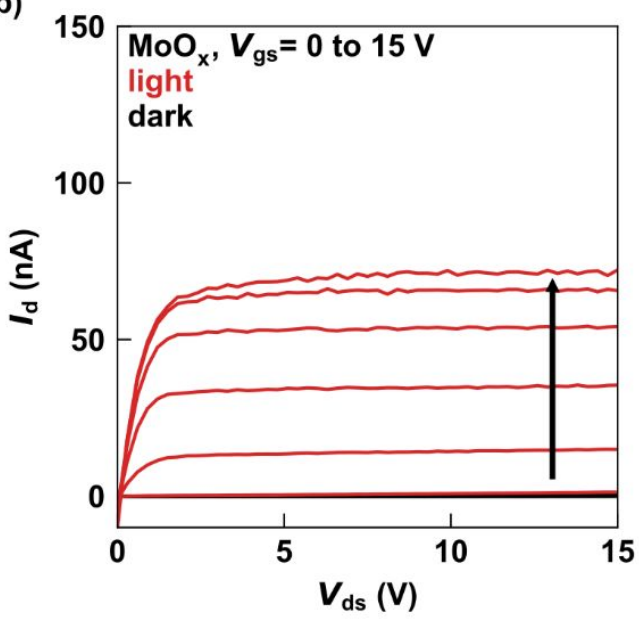

Figure. S2 The output curves for OPT with (a) Au contact and (b) $\mathrm{MoO}_{\mathrm{x}}$ contact. The colored line represents the curve measured under light and the black curve represents the curve measured under dark. 


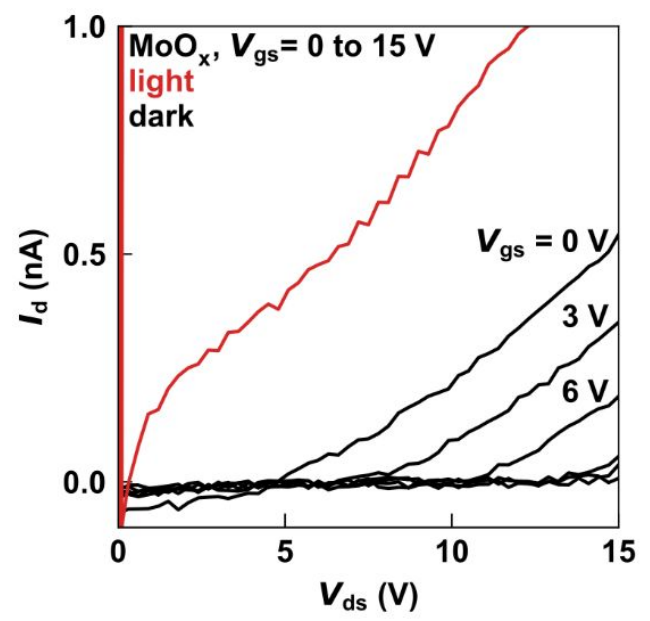

Figure. S3 The output curves for OPT with $\mathrm{MoO}_{\mathrm{x}}$ contact in larger scale. The colored line represents the curve measured under light and the black curve represents the curve measured under dark. 


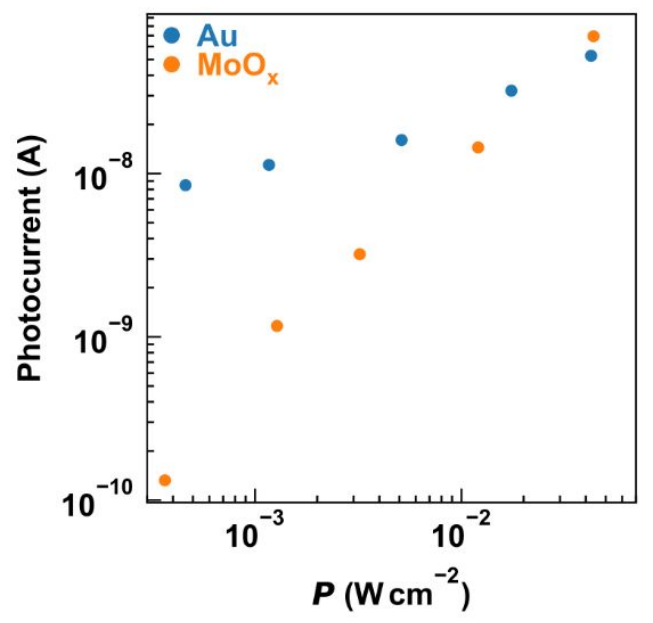

Figure S4. Photocurrent dependency as a function of light. The blue point represents the photocurrent of OPT using $\mathrm{Au}$ and the orange one using $\mathrm{MoO}_{\mathrm{x}}$. 